\title{
Fine tuning of cell behaviour by modulation of plasma membrane receptors
}

\author{
David Burnett
}

The recruitment of inflammatory cells from the blood and their activation are essential elements of the host defence in the lungs and other organs, but these processes may also have pathological consequences for' the host. Cell surface receptors are important in these cell functions in two fundamental ways. Receptors represent the senses of the cell; it is through such receptors that inflammatory cells respond to external stimuli, such as chemotactic and activating factors. Receptors in the form of adhesion molecules also allow cells to interact with each other and with connective tissues, which promotes cell to cell communication, adherence, and some movement through tissues. The in vivo environment contains a complex array of factors capable of influencing inflammatory cells, which raises the question: what determines the specificity, nature, and magnitude of cellular responses?

Undoubtedly, the composition of available ligands will to some extent influence the types of cells affected and their particular functional responses. It is, however, becoming clear that the expression of receptors on the cell surface can be modulated in ways that determine when and how the cell will respond to the presence of a ligand. Surface receptors are not static but are translocated on the plasma membrane as a cell polarises during adherence or migration. Neutrophil receptors, including those for the immunoglobulin Fc component, complement $\mathrm{C} 3 \mathrm{~b}$ (CD18/CD11b integrin), tumour necrosis factor $\alpha(\mathrm{TNF}-\alpha)$, and $N$ formyl peptides are expressed largely on the adherent or "leading" aspect of the motile cell. ${ }^{1-3}$ The distribution of receptors on the membrane may to some extent explain the exquisite sensitivity of neutrophils to substances such as the bacteria derived formylated peptides, including $N$-formy-methionylleucyl-phenylalanine (fMLP). This is a potent chemotactic factor for these cells and neutrophils are sensitive to concentration gradients of fMLP that change by only $2 \%$ over the length of the cell, ${ }^{4}$ but neutrophils nevertheless respond to concentrations varying by several orders of magnitude. The optimal concentration of fMLP for neutrophil chemotaxis is about $10^{-8} \mathrm{~mol} / 1,{ }^{5}$ but these cells can also be activated-to produce superoxide radicals, for instance-at a different optimal concentration of $10^{-6} \mathrm{~mol} / 1 .^{6}$ Ligand (fMLP) binding assays have shown that the affinity of receptors on neutrophils change in response to various stimuli and affect the cells' responses to this ligand. ${ }^{6} \mathrm{~A}$ similar phenomenon has been found with receptors for TNF- $\alpha$, which change in numbers and affinity when neutrophils adhere, in association with altered functional responses to this cytokine. ${ }^{3}$ Two explanations have been proposed to explain how one ligand, fMLP, can have fundamentally different effects on cell functions. The phenomenon has been attributed either to the expression of two different types of fMLP receptors on neutrophils, each with a different affinity for the ligand, or to a single type of receptor with interconvertible "affinity states." ${ }^{7}$ Presumably the different effects would be elicited through coupling of the receptors to alternative networks of intracellular messengers. Thus the presentation of predominantly high affinity fMLP receptors would induce chemotaxis in the event of FMLP binding, whereas low affinity receptors would lead to activation of other cell functions. ${ }^{7}$

The alternative hypotheses are both attractive but, in the absence of detailed knowledge of receptor structure, there is no conclusive evidence to support either. Evidence of interconvertible affinity states of receptors is now emerging from studies of neutrophil integrins, the family of adhesion molecules that bind to the interCellular adhesion molecules (ICAM) expressed on endothelial cells and leucocytes.

The modulation of adhesion receptors on leucocytes and "target" tissues such as endothelial cells is likely to be important in controlling the nature of recruited inflammatory cells. The expression of ICAM-1 is increased on endothelial cells, from detectable basal levels, by cytokines, including inter leukin-1 and TNF- $\alpha .^{89}$ Another endothelia1 receptor for (unidentified) neutrophil adhesion molecules, the selectin endothelial-leucocyte adhesion molecule-1 (ELAM-1), is detected on these cells only after activation. ${ }^{10}$ Thus by increasing the numbers of adhesion receptor molecules inflammatory mediators can render endothelial cells receptive to the binding of inflammatory cells. The specificity and magnitude of the inflammatory cell response also appears to be regulated by integrin adhesion molecules on leucocytes. Unlike ICAM-1 and ELAM-1, however, several factors have been shown to increase neutrophil adherence by increasing the affinity of the integrins CD18/ 
CD11a (leucocyte function associated antigen1 (LFA-1)) and CD18/CD11b (Mac-1, CR3, or Mol) without increases in the expression of receptor numbers. ${ }^{11-13}$

A mechanism for the changing affinity of the CD18/CD11a integrin has been described by Hermanowski-Vosatka and colleagues, who have identified a lipid, with a molecular weight of about $350 \mathrm{Da}$, which is known as integrin modulating factor-1 (IMF-1) and is produced by activated neutrophils. ${ }^{14}$ This lipid, yet to be fully characterised, was not detected in "resting" neutrophils but was expressed after stimulation wirth phorbol esters. It was shown to cause an increase in the affinity for complement $\mathrm{C} 3 \mathrm{bi}$, not only of cell surface CD18/CD11a but also of the isolated integrin. These results suggest that IMF-1 causes a conformational change in the integrin molecule with a resulting increase in affinity for ligand. The authors proposed that appropriate stimulation of neutrophils results in the production of IMF-1, which has the effect of increasing the affinity of cell surface integrins for ligand and consequently enhancing cell adhesion.

It is not known whether this factor acts in an autocrine or a paracrine fashion, or both. If it is secreted by the neutrophil it has the potential to affect other cells expressing the CD18/CD11a integrin. It also remains to be shown whether it is specific for this integrin or has wider effects. Possibly this lipid represents the first to be described of a family of molecules that can modulate the affinity and functions of other adhesion molecules and possibly other, unrelated receptors, such as those for fMLP and cytokines. The fact that Hermanowski-Vosatka et al have added "-1" to "IMF" suggests confidence that the identification of further related molecules will follow. Certainly the behaviour of receptors for fMLP and TNF- $\alpha$ suggests that a mechanism similar to that of IMF-1 on CD18/CD1la may be operating. The existence of such a system in neutrophils and other cells would have major implications for the control of cell functions by mediators. Molecules such as IMF-1, by modulating the receptor affinities, could orchestrate with a fine degree of sensitivity the response of a cell to a complex array of ligands. If the production of receptor modulating molecules is also up or down regulated, as suggested by the work on IMF-1, another layer of complexity and fine control is added to the system for the control of cell functions.

1 Sullivan SJ, Daukas G, Zigmond SH. Assymetric distribution of the chemotactic peptide receptor on polymorphonuclear leukocytes. J Cell Biol 1984;99:1461-7.

2 Shield JM, Haston WS. Behaviour of neutrophil leucocytes in uniform concentrations of chemotactic factors: contraction waves, cell polarity and persistence. J Cell $S c i$ tion waves, cell

3 Chamba A, Stockley RA, Burnett D. Effects of neutrophil adherence on the characteristics of receptors for tumor necrosis factor- $\alpha$. FEBS Lett 1991;282:373-6.

4 Zigmond SH. Chemotactic response of neutrophils. Am J Respir Cell Molec Biol 1989;1:451-3.

5 Senior RM, Griffin GL, Mecham RP. Chemotactic activity of elastin-derived peptides. J Clin Invest 1980;66:859-62.

6 Atkinson YH, Marasco WA, Lopez AF, Vadas MA. Recombinant human tumor necrosis factor- $\alpha$. Regulation of $N$ formyl-methionylleucylphenylalanine receptor affinity and function on human neutrophils. J Clin Invest 1988; 81:759-65.

7 Kermode JC, Freer RJ, Becker EL. The significance of functional receptor heterogeneity in the biological responses of the rabbit neutrophil to stimulation by chemotactic formyl peptides. Biochem J 1991;276:715-23.

8 Dustin M, Rothlein R, Bhhan AK, Dinarello CA, Springer TA. Induction by IL-1 and IFN- $\gamma$ : tissue distribution, biochemistry and function of a natural adherence molecule (ICAM-1). J Immunol 1986;137:245-54.

9 Pober JS, Gimbrone MA, Lopierre LA, Mendrick DL, Fiers W, Rothlein R, et al. Overlapping patterns of activation of human endothelial cells by IL-1, TNF and IFN- $\gamma$. J Immunol 1986;137:1893-6.

10 Bevilacqua MP, Stengelin S, Gimbrone MA. ELAM-1: an inducible receptor for neutrophils related to complement regulating proteins and lectins. Science 1988;243:1160-4.

11 Wright SD, Meyer BC. Phorbol esters cause sequential activation and deactivation of complement receptors on polymorphonuclear leukocytes. J Immunol 1986;136: $1759-64$.

12 Lo SK, Detmers PA, Levin SM, Wright SD. Transient adhesion of neutrophils to endothelium. J Exp Med 1989; 169:1779-93.

13 Vedder NB, Harlan JM. Increased surface expression of CD11b/CD18 (Mac-1) is not required for stimulated neutrophil adherence to cultured endothelium. J Clin neutrophil adherence
Invest 1988;81:676-82.

14 Hermanowski-Vosatka A, Van Strijp JAG, Swiggard WJ, Wright SD. Integrin modulating factor-1: a lipid that alters the function of leukocyte integrins. Cell 1992; 68:341-52. 